\title{
In-vitro antimicrobial susceptibility of Neisseria gonorrhoeae in New Zealand
}

\author{
MARGARET J. GREEN \\ From the Department of Health, National Health Institute, Wellington South, New Zealand
}

SUMMARY Four hundred and forty-two isolates of Neisseria gonorrhoeae were tested by an agar dilution method for their susceptibility to penicillin, ampicillin, tetracycline, cephaloridine, and spectinomycin. Of these isolates, 295 were tested for their susceptibility to sulphamethoxazole and to trimethoprim by the same method, using Oxoid diagnostic sensitivity test agar plus $7 \cdot 5 \%$ laked horse blood instead of Proteose No. 3 agar plus $1 \%$ IsoVitaleX and $1 \%$ haemoglobin. One hundred $(22.6 \%)$ of the isolates were found to be relatively resistant to penicillin (minimum inhibitory concentration (MIC) $>0.1 \mathrm{iu} / \mathrm{ml}$ ), but only $1.1 \%$ had a MIC of $1 \mathrm{iu} / \mathrm{ml}$ or higher. Ampicillin was slightly more active than penicillin in that all isolates were inhibited by $0.5 \mu \mathrm{g} / \mathrm{ml}$ or less. For $3.7 \%$ of isolates the MIC of tetracycline was $2 \mu \mathrm{g} / \mathrm{ml}$ or higher. All isolates were sensitive to spectinomycin. By calculating the Spearman rank correlation coefficient $\left(r_{s}\right)$, a high correlation $\left(r_{s}>0.5\right)$ was found between susceptibility to penicillin and susceptibility to ampicillin, tetracycline, and cephaloridine. Low correlation $\left(r_{s}<0 \cdot 2\right)$ was found between susceptibility to penicillin and susceptibility to spectinomycin, sulphamethoxazole, and trimethoprim.

\section{Introduction}

For years trends of the susceptibility of Neisseria gonorrhoeae to antimicrobials, especially to penicillin, have been studied in many countries (Sparling, 1972). Treatment failure associated with a decrease in the susceptibility to penicillin, and to other potentially useful antimicrobials, has been noted with concern. Recent work indicates that this trend to relative resistance may have reached a plateau in some countries (Robson and Salit, 1972; Givan and Keyl, 1974; Stolz et al., 1975; Jaffe et al., 1976). However, with the discovery of penicillinaseproducing gonococci in several countries (World Health Organisation, 1976) and reports of $N$. gonorrhoeae strains resistant to spectinomycin (Stolz et al., 1975; United States Center for Disease Control, 1977), surveillance of susceptibility seems to be even more important.

In New Zealand there has been no report of a quantitative study of the susceptibility of $N$. gonorrhoeae isolates to penicillin and other antimicrobials. This study was undertaken to provide a baseline for future surveillance.

\footnotetext{
Address for reprints: Mrs M. J. Green, Department of Health, National Health Institute, 52-62 Riddiford Street, Newtown, Wellington South, New Zealand

Received for publication 23 February 1978
}

Materials and methods

ISOLATION AND CULTURE OF

N. GONORRHOEAE

In 1976, 442 unselected isolates of $N$. gonorrhoeae were received from hospital and private medical laboratories throughout New Zealand. Of these, 251 isolates came from 20 different laboratories and the remaining 191 from a single laboratory in the South Island. The isolates, which were received in half-strength dextrose starch agar stabs overlaid with sterile oil, were kept at $35^{\circ} \mathrm{C}$ until required for antimicrobial susceptibility testing. Each isolate was then plated from the dextrose starch agar stab on to Thayer-Martin medium-GC medium base (Difco) enriched with $1 \%$ haemoglobin (Difco), $1 \%$ IsoVitaleX (Baltimore Biological Laboratories), and VCN inhibitor (Baltimore Biological Laboratories) and incubated for 48 hours at $35^{\circ} \mathrm{C}$ in a candle extinction jar. Isolates were then subcultured on to the same medium without VCN inhibitor and incubated overnight as previously.

SUSCEPTIBILITY TO ANTIMICROBIALS

Susceptibility to penicillin, ampicillin, tetracycline, spectinomycin, and cephaloridine was determined by the agar dilution method of Jaffe et al. (1976). 
The susceptibility to sulphamethoxazole and trimethoprim of 295 of the 442 isolates was determined by the same method using Oxoid DST agar and $7 \cdot 5 \%$ laked horse blood (DST medium). Of the 295 isolates, 119 were tested simultaneously using the medium of Jaffe et al. (1976) with the addition of $5 \%$ laked horse blood (Proteose medium) and DST medium. The two sets of plates were prepared from the one series of antimicrobial dilutions and were inoculated from the one set of diluted cultures.

Antimicrobial powders were obtained from: Glaxo Laboratories (NZ) Ltd (penicillin and cephaloridine); Upjohn (Australia) Pty. Ltd (tetracycline and spectinomycin); Beecham Research Laboratories (ampicillin); and Roche Products Ltd (sulphamethoxazole and trimethoprim).

Doubling dilutions over the following range of concentrations were used: penicillin $0.002-1 \cdot 0$ $\mathrm{iu} / \mathrm{ml}$; ampicillin $0.004-0.5 \mathrm{\mu g} / \mathrm{ml}$; tetracycline 0.06-4 $\mu \mathrm{g} / \mathrm{ml}$; cephaloridine $0.25-8 \mu \mathrm{g} / \mathrm{ml}$; spectinomycin 2-32 $\mu \mathrm{g} / \mathrm{ml}$; sulphamethoxazole $0 \cdot 25$ $128 \mu \mathrm{g} / \mathrm{ml}$ on DST medium and $4-128 \mu \mathrm{g} / \mathrm{ml}$ on Proteose medium; and trimethoprim 2-64 $\mu \mathrm{g} / \mathrm{ml}$ on DST medium and $8-256 \mu \mathrm{g} / \mathrm{ml}$ on Proteose medium.

Two plates containing no antimicrobials were inoculated before and after each series of antimicrobial plates and included with them in the candle extinction jars. Three control strains of $N$. gonorrhoeae with a range of known MICs for the antimicrobials tested were included with each test. These strains (F14, F18, and F29) were supplied by the US Center for Disease Control, Atlanta.

\section{CORRELATION COEFFICIENT TEST}

Spearman's rank correlation coefficient test was used to determine the correlation when MIC values of penicillin were paired with those of the other six antimicrobials tested.

\section{Results}

MIC determinations were reproducible within a two-fold limit. Figures 1, 2, 3, 4, and 5 show the distribution of the MICs of penicillin, ampicillin, tetracycline, cephaloridine, and spectinomycin for the 442 isolates of $N$. gonorrhoeae. The MICs of penicillin and ampicillin had a bimodal distribution. One hundred $(22.6 \%)$ of the isolates were found to be relatively resistant to penicillin (MIC $>0.1$ $\mathrm{iu} / \mathrm{ml}$ ), and for $1.1 \%$ the MIC was $1 \mathrm{iu} / \mathrm{ml}$ or higher. The incidence of relative resistance in the 191 isolates from the single laboratory was $20.4 \%$. All isolates were inhibited by $0.5 \mu \mathrm{g} / \mathrm{ml}$ or less of ampicillin.
The MICs of tetracycline, cephaloridine, and spectinomycin were not bimodal in distribution, but some isolates had decreased susceptibility to tetracycline and cephaloridine. For $3.7 \%$ of the isolates the MIC of tetracycline was $2 \mu \mathrm{g} / \mathrm{ml}$ or greater, and for $7.7 \%$ the MIC of cephaloridine was $8 \mu \mathrm{g} / \mathrm{ml}$ or greater. For all isolates the MIC of spectinomycin was $16 \mu \mathrm{g} / \mathrm{ml}$ or less.

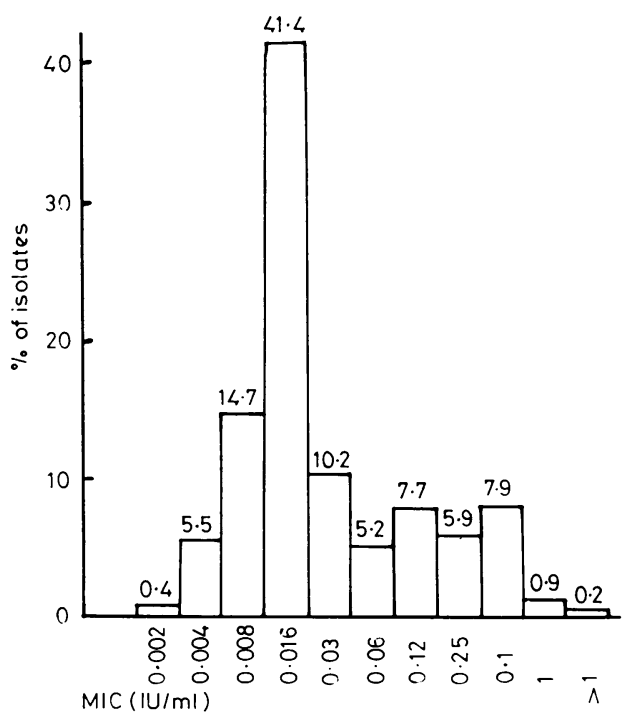

Fig. 1 MIC of penicillin for 442 isolates of N. gonorrhoeae

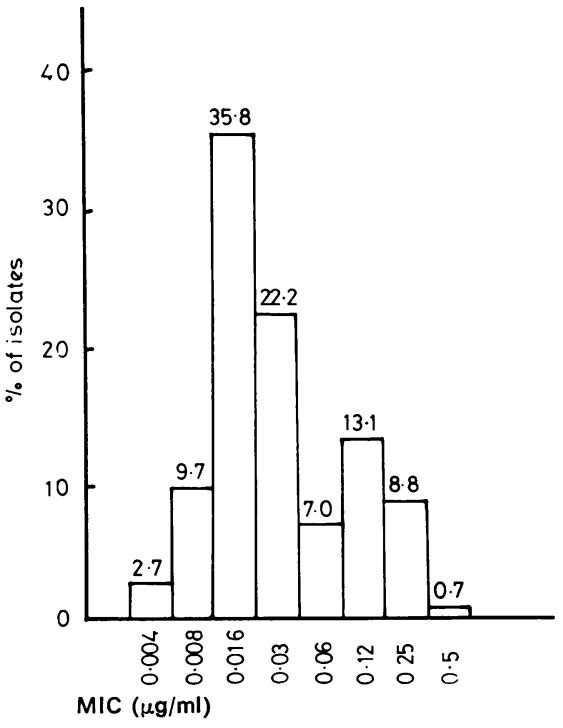

Fig. 2 MIC of ampicillin for 442 isolates of N. gonorrhoeae 
Table 1 shows the Spearman rank correlation coefficient $\left(r_{s}\right)$ for MIC values of penicillin when paired with those of ampicillin, tetracycline, cephaloridine, and spectinomycin. There is a high correlation $\left(r_{s}>0.5\right)$ between penicillin-ampicillin,

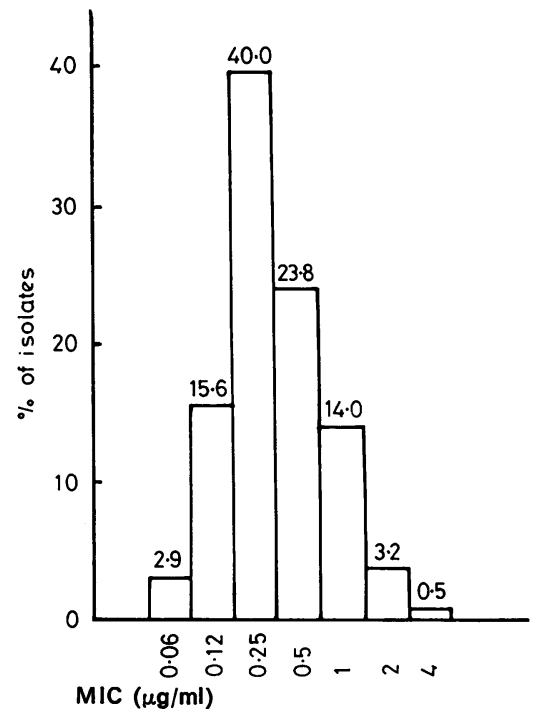

Fig. 3 MIC of tetracycline for 442 isolates of N. gonorrhoeae

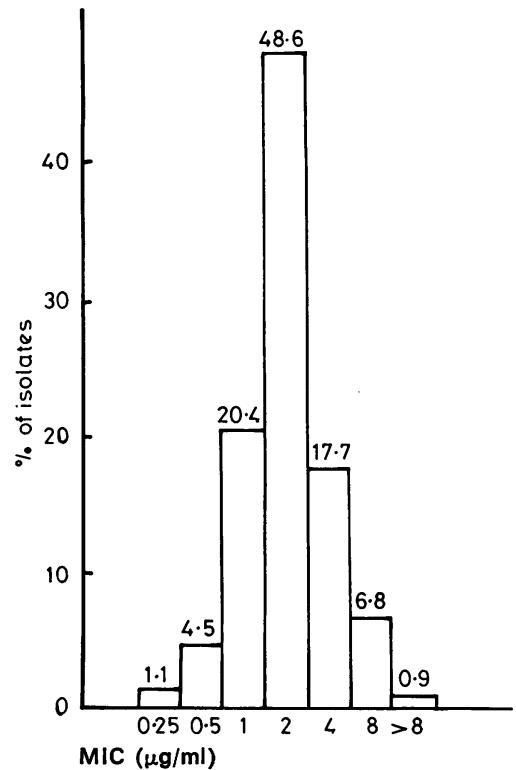

Fig. 4 MIC of cephaloridine for 442 isolates of N. gonorrhoeae penicillin-tetracycline, and penicillin-cephaloridine susceptibilities. There is a low correlation $\left(r_{s}<0 \cdot 2\right)$ between penicillin-spectinomycin susceptibilities.

Figures 6 and 7 show the distribution of MICs of sulphamethoxazole and trimethoprim for 295 isolates of $N$. gonorrhoeae. The distribution was not bimodal for either antimicrobial. The MICs of sulphamethoxazole were distributed over a wide range of concentrations, $4.4 \%$ of the isolates having a MIC of $\leqslant 0.25 \mu \mathrm{g} / \mathrm{ml}$ and $5.0 \%$ having a MIC $\geqslant 32 \mu \mathrm{g} / \mathrm{ml}$.

Table 1 Spearman rank correlation coefficients $\left(r_{\mathrm{s}}\right)$ between MIC values for 442 isolates of $\mathrm{N}$. gonorrhoeae

\begin{tabular}{ll}
\hline Antimicrobials & $r_{s^{*}}$ \\
\hline Penicillin & \\
plus ampicillin & 0.85 \\
plus tetracycline & 0.54 \\
plus cephaloridine & 0.75 \\
plus spectinomycin & 0.19 \\
All $\mathrm{r}_{\mathrm{s}}^{*}$ values are statistically significant & \\
(P $<0.001)$ & \\
\hline
\end{tabular}

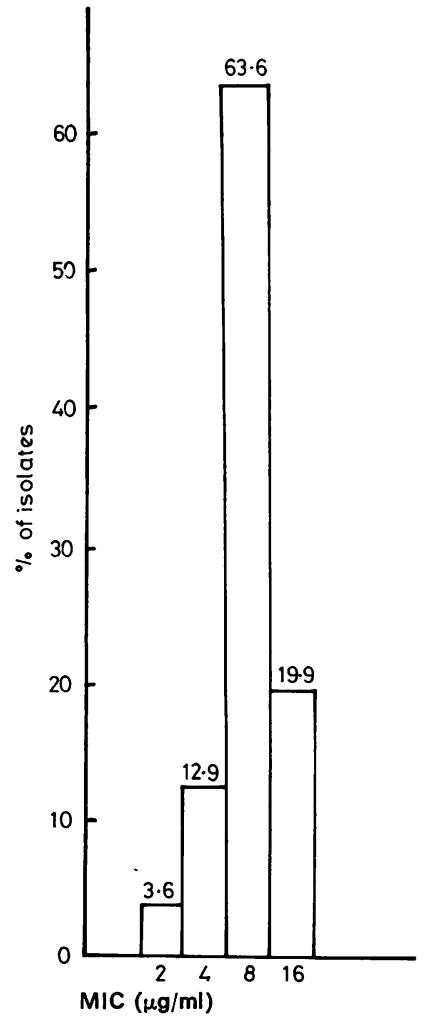

Fig. 5 MIC of spectinomycin for 442 isolates of N. gonorrhoeae 


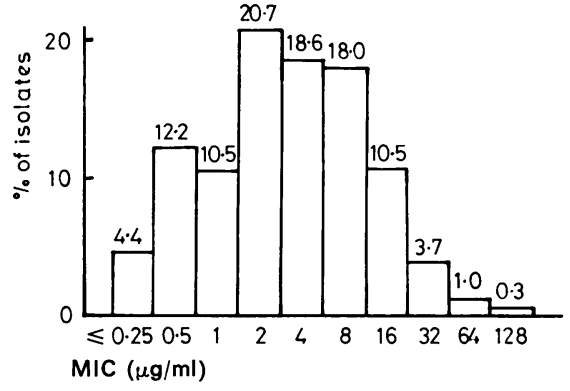

Fig. 6 MIC of sulphamethoxazole for 295 isolates of N. gonorrhoeae

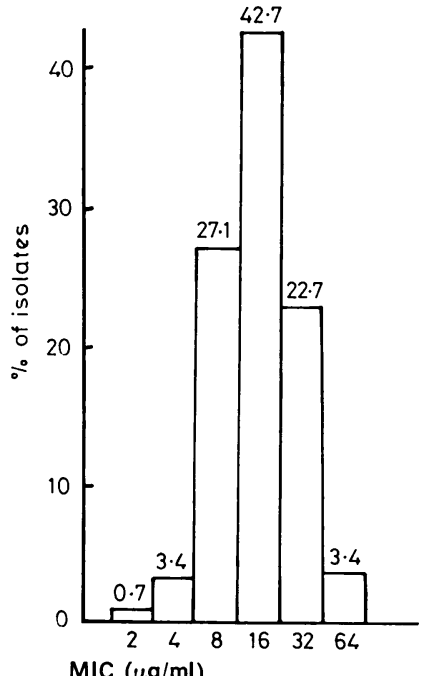

Fig. 7 MIC of trimethoprim for 295 isolates of N. gonorrhoeae

Table 2 shows the Spearman rank correlation coefficient $\left(r_{s}\right)$ when the MIC values of penicillin are paired with the values for sulphamethoxazole and trimethoprim. Low correlation $\left(r_{s} \leqslant 0 \cdot 2\right)$ is shown between penicillin susceptibility and susceptibility to sulphamethoxazole and trimethoprim.

When 119 isolates of $N$. gonorrhoeae were tested in duplicate on both Proteose and DST media, the range of MICs was approximately eight-fold higher for sulphamethoxazole and four-fold higher for trimethoprim on Proteose medium than on DST medium. The distribution of MICs on both media was similar for both antimicrobials (Figs. 8 and 9).

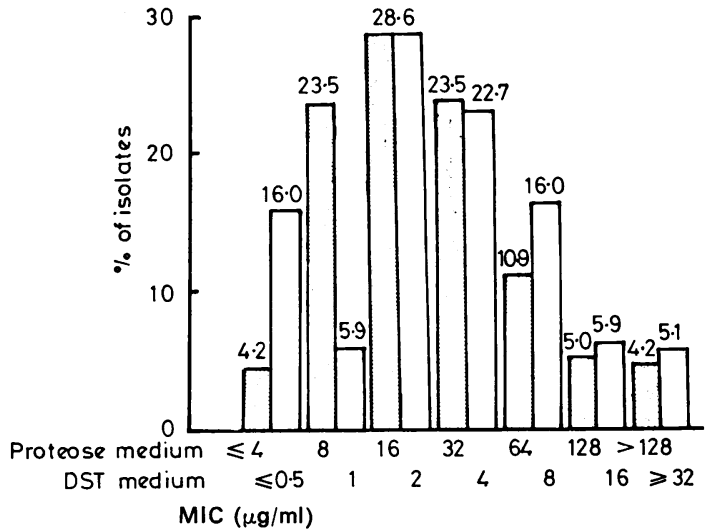

Fig. 8 MIC of sulphamethoxazole for 119 isolates of N. gonorrhoeae

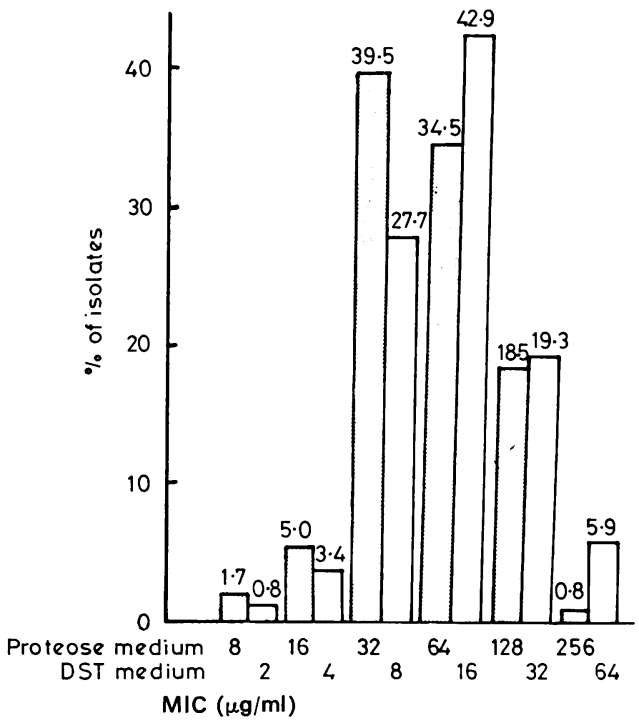

Fig. 9 MIC of trimethoprim for 119 isolates of N. gonorrhoeae

Table 2 Spearman rank correlation coefficients $\left(r_{\mathrm{s}}\right)$ between MIC values for 295 isolates of $\mathrm{N}$. gonorrhoeae

\begin{tabular}{ll}
\hline Antimicrobials & $r_{s}$ \\
\hline Penicillin & \\
$\quad$ plus sulphamethoxazole & $0 \cdot 20^{*}$ \\
plus trimethoprim & $0.08^{\dagger}$ \\
\hline
\end{tabular}

* Statistically significant $(\mathrm{P} \div 0.001)$

+ Not statistically significant $(0 \cdot 1<P<0 \cdot 2)$ 


\section{Discussion}

The incidence of relative resistance to penicillin among $N$. gonorrhoeae isolates in New Zealand appears to be similar to that in Australia and Britain but considerably lower than that in the USA. Comparisons with Australian and British studies may not be valid because of the use of different methods to test susceptibility. In Australia, Finger and Handke (1977) found $12.3 \%$ and Smithhurst (1974) found $31 \%$ of isolates had a MIC of penicillin $>0 \cdot 1 \mathrm{iu} / \mathrm{ml}$. In England, Shahidullah and Greaves (1975) found $30 \%$ of isolates had a MIC of penicillin $>0.06 \mu \mathrm{g} / \mathrm{ml}$ whereas Jaffe et al. (1976), in the USA, found $17.4 \%$ of isolates had a MIC of penicillin $>0.5 \mu \mathrm{g} / \mathrm{ml}$ and a further $51.6 \%$ had a MIC of $0.06-0.25 \mu \mathrm{g} / \mathrm{ml}$.

It has been reported (Robson and Salit, 1972; Stolz et al., 1975; Watko and Brownlow, 1975; Meheus et al., 1976; Powell and Bond, 1976) that sensitivity to penicillin is highly correlated with sensitivity to ampicillin, tetracycline, and cephaloridine. The results of this study also show this correlation.

Various workers (Stolz et al., 1975; Meheus et al., 1976; Shtibel, 1976) found no, or a low, correlation between penicillin and spectinomycin susceptibilities, and this work supports that finding also. All isolates were susceptible to spectinomycin. However, in view of recent reports of isolates which are resistant to this antimicrobial (Stolz et al., 1975; US Center for Disease Control, 1977), and its more frequent use in future to treat patients infected with penicillinase-producing gonococci, its useful life may be limited.

Stolz et al. (1975) and Meheus et al. (1976) found a relatively low correlation between penicillin susceptibility and susceptibility to sulphamethoxazole and trimethoprim. This study also shows a low correlation. Lawrence et al. (1973), however, found some correlation between the MIC of penicillin and failure of treatment with a combination of sulphamethoxazole and trimethoprim in a 20 to 1 ratio.

Testing the susceptibility of any organism to sulphonamides and trimethoprim is difficult, as the behaviour of these two antimicrobials is more medium-dependent than that of others. There is the added difficulty that gonococci require a fairly complicated medium to grow well, and growth supplements may interfere with antimicrobial action (Shtibel, 1975).

Most workers have used Oxoid DST agar with $5-10 \%$ laked horse blood and no other enrichment for testing the susceptibility of gonococci to sulphamethoxazole and trimethoprim (Phillips et al.,
1970; Lawrence et al., 1973; Stolz et al., 1975; Meheus et al., 1976); but growth on this medium was observed to be poorer than on the medium of Jaffe et al. (1976).

Yoshikawa et al. (1975) found that $95 \%$ of gonococcal strains were inhibited by $2.5 \mu \mathrm{g} / \mathrm{ml}$ trimethoprim and $47.5 \mu \mathrm{g} / \mathrm{ml}$ sulphamethoxazole in combination in Oxoid DST agar enriched with $1 \%$ IsoVitaleX and $5 \%$ laked horse blood, whereas only $84 \%$ were inhibited by the same concentrations in GC agar with the same enrichment.

The discovery of penicillinase-producing gonococci could lead to a greater evaluation of the clinical use of sulphamethoxazole combined with trimethoprim in uncomplicated gonorrhoea. It is important that a standardised method for testing susceptibility to these antimicrobials is used, so that a firm base can be provided for establishing correlations between laboratory and clinical results.

Appreciation is expressed to the staff of the laboratories who provided isolates and to the staff of the Audiovisual Aids Department, Wellington Public Hospital, who prepared the illustrations.

This paper is published with the authority of the Director-General of Health, Department of Health, Wellington, New Zealand.

\section{References}

Finger, A., and Handke, G. (1977). Antibiotic sensitivity of gonococci in South Australia 1974 to 1975. Medical Journal of Australia, 1. 133-136.

Givan, K. F., and Keyl, A. (1974). Antibiotic sensitivities of Neisseria gonorrhoeae in the Toronto area. Canadian Medical Associaton Journal, 111, 44-46.

Jaffe, H. W., Biddle, J. W., Thornsberry, C., Johnson, R. E., Kaufman, R. E., Reynolds, G. H., Wiesner, P. J., and the Cooperative Study Group (1976). National gonorrhoea therapy monitoring study. In vitro antibiotic susceptibility and its correlation with treatment results. New England Journal of Medicine, 294, 5-9.

Lawrence, A., Phillips I., and Nicol, C. (1973). Various regimens of trimethoprim-sulfamethoxazole used in the treatment of gonorrhoea. Journal of Infectious Diseases, 128 (Supplement), S673-S678.

Meheus, A., Piot, P., Pattyn, S., van Dyck, E., and vanden Berghe, D. (1976). Activity in vitro of ten antimicrobial agents against Neisseria gonorrhoeae. A study of the correlation between the sensitivities. British Journal of Venerea! Diseases, 52, 329-332.

Phillips, I., Rimmer, D., Ridley, M., Lynn, R., and Warren, C. (1970). In-vitro activity of twelve antibacterial agents against Neisseria gonorrhoeae. Lancet, 1, 263-265.

Powell, J. T., and Bond, J. H. (1976). Multiple antibiotic resistance in clinical strains of Neisseria gonorrhoeae isolated in South Carolina. Antimicrobial Agents and Chemotherapy, 10, 639-645.

Robson, H. G., and Salit, I. E. (1972). Susceptibility of Neisseria gonorrhoeae to seven antibiotics in vitro. Canadian Medical Association Journal, 107, 959-962.

Shahidullah, M., and Greaves, P. W. (1975). Minimum inhibitory concentrations of penicillin and minocycline for 300 isolates of Neisseria gonorrhoeae. British Journal of Venereal Diseases, 51, 265-266.

Shtibel, R. (1975). A new improved medium for antibiotic susceptibility testing of $N$. gonorrhoeae. Health Laboratory Science, 12, 76-81.

Shtibel, R. (1976). Resistance of Neisseria gonorrhoeae to antibacterial drugs in Ontario. Health Laboratory Science, 13, 49-53. 
Smithurst, B. A. (1974). Resistance of Neisseria gonorrhoeae to penicillin in 199 gonococcal infections in women and the response of those infections to penicillin treatment. Medical Journal of Australia, 1, 585-586.

Sparling, P. F. (1972). Antibiotic resistance in Neisseria gonorrhoeae. Medical Clinics of North America, 56, 1133-1144.

Stolz, E., Zwart, H. G. F., and Michel, M. F. (1975). Activity of eight antimicrobial agents in vitro against Neisseria gonorrhoeae. British Journal of Venereal Diseases, 51, 257-264.

United States Center for Disease Control (1977). Follow-up on antibiotic resistant Neisseria gonorrhoeae. Morbidity and Mortality Weekly Report, 26, 29-30.
Watko, L. P., and Brownlow, W. J. (1975). Antibiotic susceptibility of Neisseria gonorrhoeae isolated in the Western Pacific in 1971. British Journal of Venereal Diseases, 51, 34-37.

World Health Organisation (1976). Neisseria gonorrhoeae producing ß-lactamase (penicillinase). Weekly Epidemiological Record, 51, 385-386.

Yoshikawa, T. T., Mivamoto, S., and Guze, L. B. (1975). Comparison of in-vitro susceptibility of Neisseria gonorrhoeae to trimethoprimsulfamethoxazole on three different media. Antimicrobial Agents and Chemotherapy, 8, 515-517. 